Working Paper 92-27

June 1992
División de Economía

Universidad Carlos III de Madrid

Calle Madrid, 126

28903 Getafe

Fax: (91) 624-9849

\title{
REVISED STOCHASTIC ANALYSIS OF AN INPUT-OUTPUT MODEL
}

\author{
Thijs ten Raa and Mark F.J. Steel"
}

\begin{abstract}
A main difficulty of regional analysis is the inaccuracy of regional inputoutput data. A natural framework for investigation is stochastic input-output analysis. In his study of Central Queensland, West (1986) assumes that input coefficients are normally distributed and derives formulas for the approximation of input-output multipliers means and variances. In his normality framework, these moments do not exits, however. Moreover, an inconsistency in the derivation will be exposed. We remedy these shortcomings by respecification of the stochastic structure and by direct evaluation of the moments through Monte Carlo calculations. West's formulas are quite accurate for an aggregated version of his data set. The leading terms of the formulas can be shown to be first order approximations to the means and the variances.
\end{abstract}

Key words:

Leontief inverse, existence of moments, Monte Carlo.

" Ten Raa, Tilburg University, P.O. Box 90153, 5000 LE Tilburg, The Netherlands; Steel, Universidad Carlos III de Madrid. We thank Pieter Kop Jansen for an insightful observation and Ton Barten and an anonymous referee for helpful suggestions. The research of the authors has been made possible by senior fellowships of the Royal Netherlands Academy of Arts and Sciences. 


\section{Introduction}

Input-output analysis builds on a square, nonnegative matrix of input coefficients, A. Each column of A lists the input requirements per unit of output of a particular sector. The main tool of impact analysis is the so called Leontief inverse, $(I-A)^{-1}$, the matrix of input-output multipliers of changes in final demand into levels of outputs. West (1986) investigates the sensitivity of the input-output multipliers with respect to the input coefficients, which are not known very accurately in a regional setting. Assuming that the A matrix has normally distributed errors with zero mean and known variance, he derives formulas for the approximation of the mean and the variance of $(I-A)^{-1}$, as well as for its confidence intervals.

We will question the interpretation of this result by showing that the moments of the Leontief inverse do not exist under West's normality assumption. We will attempt to salvage his formulas by respecifying the error distribution in a way that is more in the spirit of input-output analysis and consistent with the theoretical notions of means and variances.

In deterministic input-output analysis, input coefficients are such that the Leontief inverse is nonnegative as well as $A$ itself. If the A matrix is in value terms, column sums represent total input costs per unit of output and must, for economies with value added, be less than unity. This condition is a special case of the Hawkins-Simon (1949) conditions which are necessary and sufficient for nonnegativity of the inverse. Basically, they give an explicit account of the spectral radius of $A$ being less than unity. In the hairline case of these conditions, I - A turns singular and the Leontief inverse goes off to infinity. By assuming that $A$ has a normal distribution, I - A can take all values, including singular ones, and infinite values of the Leontief inverse are not excluded. We will show that this fact destroys the existence of the means and the variances of the multipliers, depriving approximations of a foundation.

To avoid the existence problem of the moments, it is natural to confine input coefficients to the unit interval. We do so by imposing a Beta distribution on the input coefficients with the same means and variances as before. In other words, we rectify West's (1986) sensitivity analysis 
to the extent that we adjust the nature of the error distribution. We maintain its location and spread, but we prevent the tail from creeping into the singular region where Leontief inverse moments do not exist. Then we calculate the means, variances and confidence intervals of the multipliers, and contrast them with West's (1986) expressions.

In principle, we could calculate the moments by adapting West's formulas to the Beta distribution. However, since we criticize them not only on the above grounds, but also for reasons of inconsistency of derivation which will be exposed below, we rather conduct Monte Carlo computations. Notwithstanding the theoretical flaws in West's (1986) formulas, our results will confirm them for an aggregated version of his data set.

\section{Input-output multipliers formulas}

The heart of the matter described above is independent of the dimension of the model and, therefore, best addressed in the context of a single-sector economy. Input coefficients collapse into a single scalar, $\tilde{a}$, distributed normally about $a$, with some standard error, $\sigma$. The so called observed Leontief inverse is denoted $b=(1-a)^{-1}$, following West (1986, p. 365). It is not equal to the mean of the Leontief inverse defined by $\tilde{b}=(1-\tilde{a})^{-1}$, where the difference is due to the nonlinearity of the inversion. This difference is the bias induced by ignoring the stochastics. The multiplier density, moments and confidence interval can be derived from the normal density of $\tilde{a}-a,\left(2 \pi \sigma^{2}\right)^{-\frac{1}{2}} \exp \left[\frac{-1}{2 \sigma^{2}}(\tilde{a}-a)^{2}\right]$, and applying the transformation of the Leontief inverse, $\vec{b}=(1-\tilde{a})^{-1}$. Since $1-\tilde{a}=\tilde{b}^{-1}$, the Jacobian is $\tilde{b}^{-2}$ and $\tilde{a}-a=-\tilde{b}^{-1}+b^{-1}$. This yields the following density of $\tilde{b}-b$,

$$
\left(2 \pi \sigma^{2}\right)^{-\frac{1}{2}} \exp \left\{\frac{-1}{2 \sigma^{2}}\left(\tilde{b}^{-1}-b^{-1}\right)^{2}\right] \text {. }
$$

This agrees with (2.1) of West (1986), by substitution of his implicitly defined $y=\tilde{b}-b$ and, in his notation (only with superscripts * added to avoid confusion),

$$
A^{*}=b^{4} \sigma^{2}
$$




$$
\begin{aligned}
& B^{*}=b^{3} \sigma^{2} \\
& C^{*}=b^{2} \sigma^{2} .
\end{aligned}
$$

West (1986) then approximates the mean and variance of $\tilde{b}-b$ by

$$
\begin{aligned}
& E(\tilde{b}-b) \doteq \frac{b^{3} \sigma^{2}}{\left(1-7 b^{2} \sigma^{2}\right)^{3 / 7}} \\
& V(\tilde{b}-b)=V(\tilde{b}) \doteq b^{4} \sigma^{2}\left(1+\frac{59}{16} b^{2} \sigma^{2}\right)^{128 / 59} .
\end{aligned}
$$

To calculate confidence intervals for $\tilde{b}-b$, the transformation can be seen as

$$
\tilde{b}-b=\frac{1}{1-\tilde{a}}-\frac{1}{1-a}=\frac{\tilde{a}-a}{(1-a)(1-\tilde{a})}=\frac{b^{2}(\tilde{a}-a)}{b(1-\tilde{a})}=\frac{b^{2}(\tilde{a}-a)}{1-b(\tilde{a}-a)}
$$

where the last equality rests on the fact that $b=1+b a$, which can be derived from the definition, $b=(1-a)^{-1}$. $\tilde{b}-b$ is a monotonic transformation of $\tilde{a}-a$ provided that the denominator remains positive. The latter condition is fulfilled if $\tilde{a}<1$, using $b>0$ (since $a<1$ ) and the next to last expression of the above string of equalities. Consequently, any $(1-\alpha)$ confidence interval for the input coefficient, $\left[a-z_{\alpha / 2^{\sigma}}, a+z_{\alpha / 2^{\sigma}}\right]$,

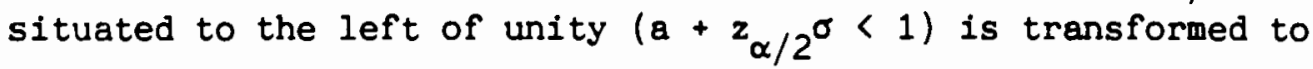

$$
\left[b-\frac{b^{2} z_{\alpha / 2^{\sigma}}}{1+b z_{\alpha / 2} \sigma}, b+\frac{b^{2} z_{\alpha / 2} \sigma}{1-b z_{\alpha / 2} \sigma}\right] .
$$

This agrees with (2.7) of West (1986), by substitution of (2.2) and (2.3). The provision $a+z_{\alpha / 2^{\sigma}}<1$ must hold for column totals in the multisector case, where West's formulas involve sums over the sectors. We shall see in section 5 that the approximations become quite useless if the latter condition is no longer satisfied. 


\section{Critique}

The density of the Leontief inverse, in deviation from the observed one, (2.1), behaves like $\tilde{b}^{-2}$ for $\tilde{b} \rightarrow \pm \infty$. This is perfectly integrable, as it should be for any proper density function. It admits no moments, however. The first moment has a density which behaves like $\widetilde{b b}^{-2}=\tilde{b}^{-1}$ and the second moment has a density which behaves like $\tilde{b}^{2} \tilde{b}^{-2}=1$, both for $\tilde{b} \rightarrow \pm \infty$. Neither expression is summable. Consequently, the moments diverge. The mean and the variance of the Leontief inverse do not exist under the assumption of normality.

A general result regarding the existence of such, so called negative moments is given in Lehmann and Shaffer (1988), who prove that if the underlying probability density function (pdf) fulfills $0<p(0+)<\infty$, the pdf of the inverse will have Cauchy tails, and will thus allow no moments for the inverse. A necessary, though not sufficient, condition for negative moments is that $p(0+)=0$. They also show that the pdf of the inverse will of ten be (at least) bimodal if negative values are not excluded. The normal distribution, in particular, suffers from nonexistence of negative moments as well as bimodality of the pdf for the inverse.

If we restrict ourselves to continuous pdf's, say $p(x)$, on the positive real line, Piegorsch and Casella (1985) find that if $\lim _{x \rightarrow 0} p(x) / x^{\alpha}<\infty$ for some $\alpha>0$, then the inverse $x^{-1}$ admits a finite mean, showing also that $p(0+)=0$ in itself is not sufficient.

Basically, the Leontief inverse goes off to infinity (plus of minus) when the input coefficient becomes unity. This value is excluded in deterministic input-output analysis, for example by Hawkins and Simon (1949), but not by West (1986). The normal distribution attaches positive mass to any neighborhood of unity. Any distribution with this property prevents existence of the moments of the Leontief inverse. West's (1986) mean and variance formulas, $(2.5)$ and (2.6), approximate moments that do not exist. At best, they are approximations to infinity.

The density formula itself, (2.1), is correct for single-sector economies. "Under the assumptions noted previously," a multi-dimensional version holds according to West (1986, p. 373). These assumptions are 
$\delta\left(a_{q s}\right) \delta\left(a_{r m}\right)=\delta\left(a_{q m}\right) \delta\left(a_{r s}\right)$ for all q, s, r, m, where $\delta\left(a_{i j}\right)$ is the $(i, j)$ th element of $\tilde{A}-A, \tilde{A}$ being the random matrix of input coefficients distributed about $A$.

(II) $\quad \delta\left(a_{i j}\right) \sim N\left(0, \sigma_{i j}^{2}\right)$

Pieter Kop Jansen observed that by the first assumption, the rank of the matrix $\tilde{A}$ - A is at most one. Typically, the error structure must be proportional for all sectors of the economy. This rules out independence of errors. Therefore, assumption $I$ is inconsistent with the independence implicit in assumption II. Thus, West's claim that the density formula holds for generic input coefficients and standard errors is based on inconsistent assumptions.

In addition, the assumption $A^{*} C^{*}=B^{*}$, given by $(2.2-4)$ for the onedimensional case, on which West's approximation of confidence intervals is founded, is generally no longer valid with more than one sector.

In short, West's (1986) formulas do not hold for multi-sector economies and his stochastic assumptions admit no mean or variance, not even for single-sector economies. Note that, in the absence of a mean, West's (1986, p. 365) claim that observed multiplier values, though biased, should be consistent, is not meaningful.

\section{Alternative stochastics}

In this section we examine an alternative stochastic structure for the input coefficients, that does not preclude the existence of moments for the multipliers. This is achieved by restricting the support of the pdf on $\tilde{a}$ to the unit interval, on which we specify a Beta density.

Thus, in the single-sector case, $\tilde{a} \sim \beta(p, q)$ with pdf

$$
B(p, q)^{-1} \tilde{a}^{p-1}(1-\tilde{a})^{q-1}, 0 \leq \tilde{a} \leq 1 \text { and } p, q>1
$$

The last inequality is necessary and sufficient for unimodality; see, e.g. Johnson and Kotz (1970, p. 41). Note that $q>1$ implies that $\lim p(1-\tilde{a}) /(1-\tilde{a})^{\alpha}=0$, so that the first negative moment should then $\tilde{\mathbf{a}} \uparrow 1$ 
exist, according to Piegorsch and Casella (1985). $\tilde{b}=(1-\tilde{a})^{-1}$ has the following density.

$$
B(p, q)^{-1}\left(\tilde{b}^{-1}\right)^{q-1}\left(1-\tilde{b}^{-1}\right)^{p-1} \tilde{b}^{-2}=B(p, q)^{-1} \tilde{b}^{-q-1}\left(1-\tilde{b}^{-1}\right)^{p-1}
$$

from which its $r-t h$ moment has a density which behaves like $\left(1-\tilde{b}^{-1}\right)^{p-1}$ for $\tilde{b} \rightarrow 1$ and $\tilde{b}^{-q-1+r}$ for $\tilde{b} \rightarrow \infty$. It is integrable if $-q-1+r<-1$. (The part near $\tilde{b}=1$ is automatically integrable by $p \geq 1$.) Since we want at least two moments, we need $q>2$.

Parameters $p$ and $q$ are determined by the observed mean and standard error of the input coefficient. The moments of $\tilde{a}$ are easily evaluated and equated with a and $\sigma^{2}: \frac{p}{p+q}=a$ and $\frac{p q}{(p+q)^{2}(p+q+1)}=\sigma^{2}$.

This is obtained by putting

$$
p=a\left(\frac{a-a^{2}}{\sigma^{2}}-1\right) \text { and } q=(1-a)\left(\frac{a-a^{2}}{\sigma^{2}}-1\right) \text {. }
$$

They are bigger than 1 and 2, respectively, if and only if $\sigma^{2}$ is small enough, more precisely, if and only if $\sigma^{2} \leq a \frac{a-a^{2}}{1+a}$ and $\sigma^{2}<a \frac{(1-a)^{2}}{3-a}$. respectively. It is easy to see that the first constraint dominates the second on $0 \leq a \leq 1 / 3$ and that, there, a sufficient condition is $\sigma / a \leq \sqrt{1 / 2}$, which is tight at $a=\frac{1}{3}$. On $1 / 3 \leq a \leq 1 / 2$ the second constraint dominates and a sufficient condition is $\sigma / a<\sqrt{1 / 5}$, which is tight at $a=\frac{1}{2}$. On $\frac{1}{2}<a<1$, the second constraint continues to dominate and drives $\sigma^{2}$ down to zero when a approaches unity.

Although West's formulas, (2.5) and (2.6), are theoretically flawed, they and generate results which are close to our Monte Carlo estimates, as we shall see shortly. Let us also compare them to the theoretical results in our present setting. Since $\tilde{b}$ has its density given by (4.1), it is straightforward to derive the mean,

$$
E(\tilde{b})=B(p, q)^{-1} B(p, q-1)=\frac{p+q-1}{q-1}
$$

Substituting $(4.2)$ and $b=(1-a)^{-1}$ and subtracting $b$, we obtain 


$$
E(\tilde{b}-b)=\frac{b^{3} \sigma^{2}}{1-\frac{b+1}{b-1} b^{2} \sigma^{2}}
$$

Similarly, we derive

$$
\begin{aligned}
& E\left(\tilde{b}^{2}\right)=B(p, q)^{-1} B(p, q-2)=\frac{(p+q-1)(p+q-2)}{(q-1)} \text { and } \\
& V(\tilde{b}-b)=V(\tilde{b})=b^{4} \sigma^{2} \frac{\left[1-\frac{b^{2} \sigma^{2}}{b-1}\right]\left[1-\frac{2 b^{2} \sigma^{2}}{b-1}\right]}{\left[1-\frac{b+1}{b-1} b^{2} \sigma^{2}\right]^{2}\left[1-\frac{2 b+1}{b-1} b^{2} \sigma^{2}\right]} .
\end{aligned}
$$

West's approximation formulas, (2.5) and (2.6), and our exact formulas, (4.3) and (4.4), are close for $b^{2} \sigma^{2}$ small. Then $E(\tilde{b}-b)$ tends to $b^{3} \sigma^{2}$. both in (2.5) and in (4.3), and $V(\tilde{b}-b)$ tends to $b^{4} \sigma^{2}$, both in $(2.6)$ and in (4.4). Note that the stochastic assumptions underlying both sets of formulas are different, however.

\section{A Monte Carlo experiment}

Although the previous section contains explicit expressions for the moments of the multiplier in a single-sector economy using a Beta distribution, the extension to $n>1$ sectors defies exact analytical treatment. To avoid the kind of inconsistencies that West (1986) had to use (see our Section 3), we perform a Monte Carlo experiment, where the input coefficients are all drawn from independent Beta distributions with $p$ and $q$ chosen as in (4.2) for each element of A. Rejection of those drawings where the Hawkins-Simon conditions are violated ensures invertibility of Leontief matrices in the multi-sector case. (Our stochastic assumptions in section 4 only guarantee existence of moments for $n=1$.) In addition, a Monte Carlo approach allows us to evaluate any function of A we wish to consider, and directly provides us with full density plots.

West (1986) uses his formulas to examine multipliers, more precisely, their means, standard errors and confidence intervals. To define the multipliers, let the last sector be households and consider any of the other sectors, k. The disaggregated multipliers of this sector are listed in the $\mathbf{k - t h}$ column of the Leontief inverse. The simple sum of all these disaggregated multipliers but the last one defines the output multiplier of sector 
k. If the terms are weighted by the households income row coefficients (the bottom row of the full A matrix), one obtains the so called income multiplier of sector $k$. If the weights are employment coefficients, one obtains the employment multiplier of sector $\mathbf{k}$.

Since West (1986) contains no data, we turn to West (1982), where we find aggregated input-output data, reproduced in tables 1 and 2 . Since employment figures are missing, we confine ourselves to output and income multipliers. The output multipliers are the column totals of the Leontief inverse of the full system, excepting the bottom entries. It can be shown that these bottom entries match the income multipliers, if the household sector has zero income.

We check West's formulas (with summation over all $n+1=6$ sectors) by Monte Carlo estimates of the means, standard errors and confidence intervals of the output and income multipliers under our specification of the error distribution, chosen to obtain theoretical consistency. In particular, we assume independent Beta distributions on the unit interval for the input coefficients with West's (1982) means and standard deviations. Using the original data from Tables 1 and 2, we observed that West's formulas and our Monte Carlo estimates (using 20,000 drawings) yielded very similar means, standard errors and confidence intervals. The results are given in table 3. Relative to our Monte Carlo results. West's (1986) formulas are accurate for the means of the multipliers to the third decimal and get standard errors and confidence interval borders right to the second decimal. In other words, even though West's formulas are proxies to moments which do not exist and the derivation is inconsistent in the multi-dimensional case, they perform well in our context which admits theoretical values of the moments and uses direct Monte Carlo calculations. Both estimates of the mean are larger than the observed values, corresponding to Simonovits' (1975) proof of this inequality for any distribution on $\widetilde{A}$ under independence.

\section{A first order analysis}

The good performance of West's formulas, despite the observed problems, is fascinating. The mono-sector case analysis (sector 4) provides some intuition. It was demonstrated that West's approximation formulas and our exact 


\section{small}

formulas collapse for $r^{2} \sigma^{2}$ into $E(\tilde{b}-b) \doteq b^{3} \sigma^{2}$ and $V(\tilde{b}-b) \doteq b^{4} \sigma^{2}$. In the multi-sector case, West's formulas tend to

$$
\begin{aligned}
& E\left[\sum_{h}^{n}\left(\tilde{b}_{h k}-b_{h k}\right)\right] \doteq \sum_{i, j}^{n} b_{j k}\left[\sum_{h}^{n} b_{h i}\right] b_{j i} \sigma_{i j}^{2} \\
& v\left[\sum_{h}^{n}\left(\tilde{b}_{h k}\right)\right] \doteq \underset{i, j}{\Sigma}\left[b_{j k}\left[\begin{array}{ll}
n \\
\Sigma & b_{h i}
\end{array}\right] \sigma_{i j}\right]^{2} .
\end{aligned}
$$

These first order approximations can be shown to hold indeed. For a detailed analysis, we refer to ten Raa and Kop Jansen (1992). To develop the Intuition, we shall provide a heuristic derivation of (6.1). From the definition of the Leontief inverses,

$$
\widetilde{B}-B=\widetilde{B}(\widetilde{A}-A) B
$$

and, therefore,

$$
\widetilde{B}-B=[B+\widetilde{B}(\tilde{A}-A) B](\widetilde{A}-A) B \text {. }
$$

Taking expectations, the first term on the right hand side drops out by definition of $A$. In the second term, a first approximation of $\widetilde{B}$ is $B$. Hence, in first approximation,

$$
E\left(\tilde{b}_{h k}-b_{h k}\right)=\sum_{\substack{i, j \\ r, s}}^{n} E\left[b_{h i}\left(\tilde{a}_{i j}-a_{i j}\right) b_{j r}\left(\tilde{a}_{r s}-a_{r s}\right) b b_{s k}\right] .
$$

By independence, only the terms with $(r, s)=(i, j)$ persist. Summation over h yields (6.1).

The first order approximations hold for small variances and independence. Normality is not needed and the refinements constituted by West's formulas are misleading for the reasons given in section 3 . 


\section{Conclusion}

From a purely conceptual point of view, we argue against the use of approximations to moments that do not exist under the assumptions made, at least when a viable alternative is available. The fact that normality of input coefficients does not admit finite moments for the elements of the Leontief inverse is felt to be very problematic if we are interested in evaluating such moments. This situation is remedied by making an alternative stochastic assumption, using the Beta distribution defined on the unit interval, which does not prevent existence of these negative moments, given certain restrictions on its parameters. In addition, the theoretical inconsistencies that West (1986) has to introduce in order to extend the analysis to a multi-sector case, lead us to adopt a Monte Carlo approach. In view of these theoretical problems, the actual numerical values of the formulas suggested by West seem very good approximations in the particular, highly aggregated, case examined by West (1982). The reason is that West assumes small variances. Under this assumption, the leading terms of his formulas can be shown to be first order approximations to the mean and the variance of the Leontief inverse. 
Table 1. Direct Input Coefficients Matrix, $a_{i j}$

\begin{tabular}{|crrrcrc|}
\hline Sector & 1 & 2 & 3 & 4 & 5 & $\mathrm{H}-\mathrm{H}$ \\
\hline 1 & 0.0885 & 0.0172 & 0.1545 & 0.0000 & 0.0007 & 0.0366 \\
2 & 0.0001 & 0.0225 & 0.0197 & 0.0014 & 0.0059 & 0.0000 \\
3 & 0.1283 & 0.1421 & 0.1927 & 0.0468 & 0.1056 & 0.2723 \\
4 & 0.0440 & 0.0731 & 0.0584 & 0.0372 & 0.0393 & 0.1600 \\
5 & 0.0425 & 0.1148 & 0.0661 & 0.1395 & 0.0775 & 0.3525 \\
$\mathrm{H}-\mathrm{H}$ & 0.0913 & 0.1300 & 0.2344 & 0.3833 & 0.4239 & 0.0000 \\
\hline
\end{tabular}

Source: West (1982).

Table 2. Input Coefficient Standard Error Matrix, $\sigma_{1 j}$

\begin{tabular}{|crrrccc|}
\hline Sector & 1 & 2 & 3 & 4 & 5 & $\mathrm{H}-\mathrm{H}$ \\
\hline 1 & 0.0048 & 0.0030 & 0.0055 & 0.0000 & 0.0001 & 0.0009 \\
2 & 0.0000 & 0.0036 & 0.0004 & 0.0001 & 0.0002 & 0.0000 \\
3 & 0.0066 & 0.0073 & 0.0079 & 0.0045 & 0.0061 & 0.0057 \\
4 & 0.0042 & 0.0064 & 0.0030 & 0.0048 & 0.0041 & 0.0059 \\
5 & 0.0058 & 0.0067 & 0.0046 & 0.0049 & 0.0021 & 0.0026 \\
$\mathrm{H}-\mathrm{H}$ & 0.0048 & 0.0031 & 0.0056 & 0.0070 & 0.0055 & 0.0000 \\
\hline
\end{tabular}

Source: West (1982). 
References

Johnson, N.L. and S. Kotz, (1970): Distributions in Statistics: Continuous Univariate Distributions 2. Boston: Houghton Mifflin.

Hawkins, D., and H.A. Simon, (1949): "Some Conditions of Macro-economic Stability," Econometrica, 17, 245-248.

Lehman, E.L., and J.P. Shaffer, (1988): "Inverted Distributions," The American Statistician, 42, 191-194.

Piegorsch, W.W., and G. Casella, (1985): "The Existence of the First Negative Moment," The Amprican Statistician, 39, 60-62.

ten Raa, Th., and P. Kop Jansen, (1992): "First Order Analysis of the Bias and Variance of Multipliers," Working Paper, Tilburg University.

Simonovits, A., (1975): "A Note on the Underestimation and Overestimation of the Leontief Inverse". Econometrica, 43, 493-498.

West, G.R., (1982): "Approximating the Moments and Distributions of InputOutput Multipliers," Working Papers in Economics 36, University of Queensland.

West, G.R., (1986): "A Stochastic Analysis of an Input-Output Model," Econometrica, 54, 363-374. 
Table 3. Multiplier Values, Moments and Confidence Intervals

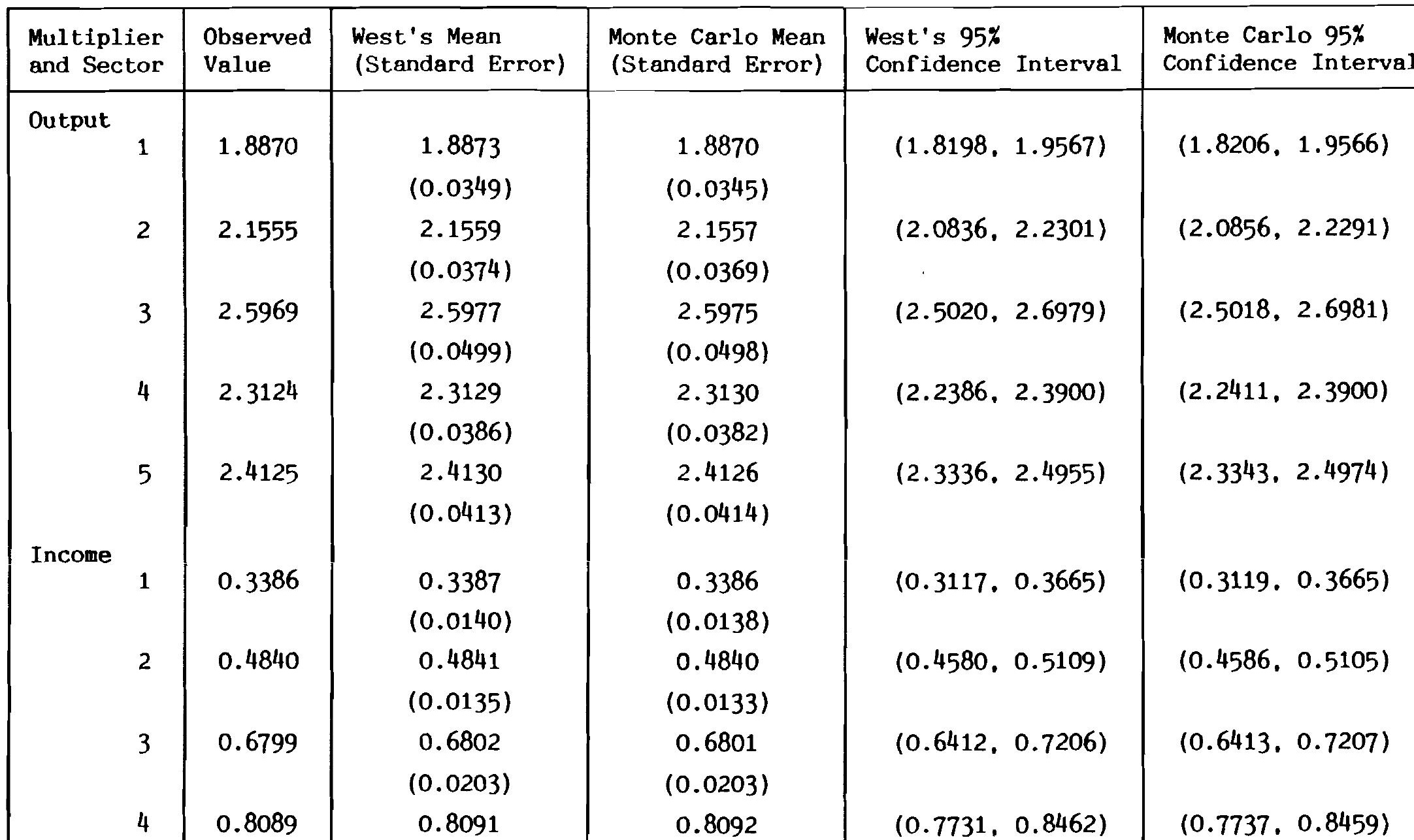

